\title{
The effects of vibration and massage on severity of symptoms of restless leg syndrome and sleep quality in hemodialysis patients; a randomized cross-over clinical trial
}

\author{
Somayeh Azimpour ${ }^{\mathbb{D}}$, Habibollah S Hosseini ${ }^{2}$, Adel Eftekhari ${ }^{3}$, Majid Kazemi $^{*{ }^{*}}$ \\ ${ }^{1}$ School of Nursing and Midwifery, Rafsanjan University of Medical Sciences, Rafsanjan, Iran \\ ${ }^{2}$ Nursing Department, Nursing and Midwifery School, Geriatric Care Research Center, Rafsanjan University of Medical Sciences, \\ Rafsanjan,Iran \\ ${ }^{3}$ Department of Health in Disasters and Emergencies, School of Public Health, Shahid Sadoughi University of Medical Sciences, Yazd, Iran \\ ${ }^{4}$ Department of Medical Surgical Nursing, Faculty of Nursing and Midwifery, Non-Communicable Disease Research Center, Rafsanjan \\ University of Medical Sciences, Rafsanjan, Iran
}

\section{A R T I C L E I N F O}

Article Type:

Original

\section{Article History:}

Received: 10 September 2018

Accepted: 6 December 2018

Published online: 14 December 2018

\section{Keywords:}

Restless leg syndrome

Sleep quality

Hemodialysis

End-stage renal disease

\begin{abstract}
A B S T R A C T
Introduction: Restless leg syndrome (RLS) is a common sensory disturbance in hemodialysis patients causing mental disorders, sleep disturbances, and other problems.

Objectives: This study compared the effects of massage and vibration on sleep quality and severity of symptoms of RLS in hemodialysis patients.

Patients and Methods: This cross-over clinical trial was conducted on 80 hemodialysis patients with RLS who were assigned into two groups randomly. The first group received massage and the second group received vibration. Each group received the related intervention for one month three times per week each session lasting 10 minutes. One month after the first intervention, the interventions were changed in a cross-over design between the two groups. The severity of symptoms of RLS and sleep quality were assessed before and after interventions using the related questionnaire.

Results: The means of severity of symptoms of RLS and sleep quality showed a significant improvement after both interventions of vibration and massage $(P<0.001)$. Additionally, a significant difference was observed in the means of severity of RLS and sleep quality after interventions between two groups hence vibration exerted a greater effect on improving the symptoms of RLS and sleep quality in patients compared to massage $(P=0.001)$.

Conclusion: Our findings suggested that both vibration and massage reduce the mean of severity of RLS and improve sleep quality in hemodialysis patients, with vibration exerting a greater effect compared to massage.
\end{abstract}

Implication for health policy/practice/research/medical education:

In a study on 80 hemodialysis patients, we found vibration and massage therapy significantly reduced the mean intensity of restless leg syndrome symptoms and improved sleep quality in hemodialysis patients.

Please cite this paper as: Azimpour S, Hosseini HS, Kazemi M, Eftekhari A. The effects of vibration and massage on severity of symptoms of restless leg syndrome and sleep quality in hemodialysis patients; a randomized cross-over clinical trial. J Renal Inj Prev. 2019;8(2):106-111. DOI: 10.15171/jrip.2019.20.

Introduction

The end-stage of renal failure involves the irreversible and progressive reduction of kidney function which requires alternative treatment for kidney function (1). Currently, the most common treatment modality for pronounced renal failure around the world is hemodialysis (2). Although hemodialysis has caused longer survival of hundreds of patients among thousands of end-stage renal disease (ESRD) patients, they are exposed to various problems and complications (3). Among them, nervous system complications, burning sensation in the body, restless leg syndrome (RLS), feet prolapse (podiatric ptosis), and even 
panplegia are examples for hemodialysis complications (4). RLS is one of the common complications among ESRD patients (5). It is a neurologic sensory-motor impairment (6) which is described as an unpleasant feeling in the feet occurring as a result of inclination for moving the lower extremities and relieves with movement (1). In the case of aggravation of symptoms, this condition can involve the arms and other parts of the body (7). These symptoms are usually manifested at night or at rest leading to sleep disturbances (1). On the basis of international standards of RLS, the diagnostic criteria of this syndrome are as follows: 1) Sensory symptoms in the feet leading to frequent uncontrollable movements in the feet, 2) Onset or deterioration of symptoms at rest, 3) Improvement or relief of symptoms at rest, and 4) Aggravation of symptoms in the evening or at night. Patients describe these unpleasant feelings as stinging, crawling of an organism on the skin, itching of the lower extremities, flow of water in the feet, feeling of presence of insects in the bone, or current flow in the feet (8). Almost $20 \%-80 \%$ of hemodialysis patients experience this syndrome while its prevalence in the public community is $2 \%-15 \%$. In the study by Wong et al, $70 \%$ of the hemodialysis patients suffered from RLS (9). This syndrome can induce problems like insomnia, daily drowsiness, bad quality of life, and depression (7). It is one of the most important factors in creating sleep disorders in renal failure patients under hemodialysis which, according to present literature, affects $62 \%$ of these patients (10). The treatment of this syndrome includes both pharmaceutical and non-pharmaceutical therapies. Treatment depends on the severity and frequency of the symptoms. Mild forms of this syndrome may be managed by changing the life style and the severe forms may be treated by pharmaceutical therapy (11). Considering the numerous medicines used for treatment and the unexpected complications of these drugs in the patients, the provision of non-pharmaceutical treatment methods for these patients seems mandatory. The nonpharmaceutical factors recommended including changing the life style such as avoiding intake of caffeine or alcohol before going to bed, music therapy methods, palpation therapy (acupressure), cryotherapy, the application of heat, acupuncture, induction of relaxation in the patient (psychological suggestion), hypnosis, massage therapy, progressive muscular relaxation, and yoga are effective in controlling the severity of this syndrome (12). Some studies have demonstrated that vibration can increase flexibility along with increased maximal muscular power (13). Another potential effect of vibration is its ability to change histologic perfusion and modulation of vascular network (matrix) resulting in increased blood circulation in the body (14). Vibration increases vascular dilatation, enhances superficial and deep blood flow, and increases muscular temperature leading ultimately to decreased tissue viscosity and increased muscular elasticity. On the other hand, reduced pain after application of vibration can predispose to increased ROM (range of motion) and facilitate flexibility through increasing pain threshold (15). Mitchell and Johnson showed in their study the effect of vibration on increasing the blood stream in RLS (16). Additionally, Doyle et al stated that whole body vibration before hemodialysis is acceptable and safe and is associated with improved physical function (17). Massage therapy is another non-pharmaceutical treatment for RLS. It increases plasma serotonin level which probably inhibits the transmission of harmful signals to the brain. Massage causes comfortable sleep leading to reduced pain sensation since somatostatin is normally released during deep sleep without which pain is felt. Hence, when individuals are deprived of deep sleep, somatostatin may be released to a lesser amount (18). Moreover, massage increases peripheral blood circulation, increased mechanical movement of fluids in the veins, lymphatic vessels, and blood vessels (19). Another study investigated the effect of massage of lower and upper back and feet on fatigue and sleep quality in hemodialysis patients. A biweekly onemonth massage program resulted in diminished fatigue and improved sleep quality in the patients (20). Although pharmaceutical therapies are common for treating RLS, considering the concerns with the complications associated with drug therapy, the researchers are seeking complementary treatments which are cost-effective with little complications.

\section{Objectives}

Massage therapy and vibration are two complementary therapeutic methods many useful effects of which have been proved. However, no study has yet compared the effects of massage therapy and vibration on the severity of RLS and sleep quality. Therefore, this study aimed at comparing the efficacy of massage therapy and vibration in relieving the intensity of RLS symptoms and improving sleep quality in hemodialysis patients.

\section{Patients and Methods \\ Study population}

This was a randomized cross-over clinical trial conducted on 80 hemodialysis patients who were selected with purposive sampling method from among the hemodialysis patients presenting to three hospitals in Meibod, Ardakan, and Yazd in Iran and assigned randomly into two groups. Four samples were excluded from the study due to lack of their cooperation, so, the study was carried out on 76 patients. The inclusion criteria were; passage of at least three months after hemodialysis, presence of RLS (a score of 11+), absence of consciousness impairment, enjoying proper neurological, skeletal, and vascular status, and absence of any malignancy, ulcer, and dermal erythamosis. In addition, the exclusion criteria were; intake of analgesics, drug abuse, taking of psychiatric and neurologic medicines, lack of inclination for participation, and the use of vibration and massage at 
home. The research goals, procedures, and stages were explained completely to the participants while informed written consent was obtained from each patient. The data were collected by one of the researchers by interviews using RLS International Questionnaire and Pittsburgh Sleep Quality Index. First, the patients were selected with RLS International Questionnaire. This inventory included ten 5 -point items ranging between 0 and 4 points with the total score of the tool ranging between 0 and 40 . The severity of the disorder was divided into five classes on the basis of the obtained scores including without problem $=0$, mild problem $=1-10$, severe problem $=21-30$, and very severe problem $=31-40$. The individuals who scored $11+$ entered the study. The content validity of the questionnaire was verified scientifically and its reliability was established by Molahosseini et al as 95\% (21). The patients were randomly assigned into two groups of 40 (vibration group and massage group). First, the researcher completed the demographic information questionnaire including age, gender, occupation, education level, history of dialysis, pain relief methods, and analgesics intake and also completed the Pittsburgh Sleep Quality Index that measured sleep quality during one month regardless of the patient's opinion. This questionnaire includes seven categories including the patient's general description of sleep quality, latency in going to sleep, duration of useful sleep, the proportion of useful sleep time to the total time spent in the bed, sleep disorder, awakening due to dyspnea or apnea, nocturnal cough, limbs pain, extreme cold, extreme heat, the use of narcotic drugs for going to sleep, drowsiness, and lack of interest for doing ADL (activity of daily living) due to impaired sleep. Each scale of the tool receives a score between 0 and 3 . A total score of 5 or more indicates low sleep quality and a score less than 5 demonstrates high quality of sleep. Farrahi et al obtained sensitivity of $100 \%$, specificity of $93 \%$, and Cronbach's a of $89 \%$ for the Persian version of this questionnaire (22). In group $\mathrm{A}$, the patients underwent massage therapy for one month at the time of dialysis. Massaging was performed by a trained person three times per week each session lasting 10 minutes using hacking massage in both feet. This method uses sequential rapid hacking massage without pauses with the side of the hands using two modalities.

\section{The first modality}

Longitudinal; both hands move together along the length of the muscle.

\section{The second modality}

Convergent-divergent method; both hands are placed on the superior and inferior surfaces of the muscle converging to each other with hacking massage and then diverge. In group $\mathrm{B}$, the patients underwent vibration therapy for one month on both feet three sessions per week each session lasting 10 minutes at the time of hemodialysis, and vibration was conducted at low voltage (Thrive Model
717A). The severity of RLS symptoms and sleep quality were measured in both groups after a 4-week intervention. After a 4-week wash out interval (with no intervention), the message group underwent vibration therapy for one month three sessions per week in a cross-over design. In addition, the vibration group underwent massage therapy for one month three sessions per week in a cross-over design. Again, the severity of RLS symptoms and sleep quality were measured in both groups before and after interventions.

\section{Ethical issues}

This research was performed based on the Declaration of Helsinki principles. Informed written consent was obtained from each patient. All information about individuals was coded and kept confidential. This study was approved by the Committee of Ethics in Human Research at Rafsanjan University of Medical Sciences with code Ir.rums.rec.1394.193 dated 20/2/2015 and registered in Iranian Registry of Clinical Trials (IRCT) (identifier: IRCT2016022826814N1; http://www.irct.ir/trial/22121).

\section{Statistical analysis}

All data were analyzed using SPSS 18. Results are expressed as mean \pm SD or percentage. Data were analyzed using statistical tests including the $t$ test, to compare massage and vibration variables. Also findings before and after intervention in each group were analyzed using paired samples $t$ test. Additionally, $P$ values of less than 0.05 were considered statistically significant.

\section{Results}

Our findings indicated that the mean age of the samples was 61.41 years, $57.5 \%$ of the patients were male, most of the participants $(48.8 \%)$ were illiterate, and most of them (43.8\%) were unemployed. The results demonstrated that most patients were at the moderate $(68.8 \%)$ and severe $(31.3 \%)$ levels of severity of RLS symptoms before intervention, while, they were at the mild (16.3\%) and moderate $(78.8 \%)$ levels of severity of RLS symptoms after vibration therapy. Furthermore, they were at the moderate $(93.8 \%)$ and severe $(1.3 \%)$ levels of severity of RLS symptoms after massage therapy (Table 1).

Our findings also suggested that both intervention modalities exerted an effect on RLS with the mean of RLS score decreasing after both vibration and massage therapy

Table 1. Frequency distribution of severity of RLS before and after interventions.

\begin{tabular}{lccc}
\hline \multirow{2}{*}{ RLS } & Before intervention & After vibration & After massage \\
\cline { 2 - 4 } & No. (\%) & No. (\%) & No. (\%) \\
\hline Mild & $0(0)$ & $13(16.3)$ & $0(0)$ \\
Moderate & $55(68.8)$ & $63(78.8)$ & $75(93.8)$ \\
Severe & $25(31.3)$ & $0(0)$ & $1(1.3)$ \\
Total & $80(100)$ & $76(100$ & $76(100)$ \\
\hline
\end{tabular}


$(P=0.001)$. Moreover, a comparison of the two methods revealed that vibration therapy was more effective on RLS symptoms than massage therapy indicating a statistically significant difference $(P=0.001$; Table 2$)$.

Additionally, the study on the effects of massage therapy and vibration on sleep quality indicated that the mean score of sleep quality decreased after vibration and massage therapy indicating improved quality of sleep since the results of paired $t$ test indicated a significant difference in sleep quality before and after interventions $(P=0.001)$. Furthermore, in comparing two methods of massage therapy and vibration, the results of t-test suggested a significant difference in sleep quality between the two groups after interventions $(P=0.001)$ (Table 3$)$.

\section{Discussion}

Our findings indicated that the mean score of RLS symptoms decreased after vibration therapy in the study population (12.82 versus 18.99). Yoosefinejad et al showed that vibration affects the muscular power and balance in type II diabetic patients with neuropathy (23). Moreover, Junggi reported reduced pain and improved balance and gait coordination in type 2 diabetic patients with peripheral neuropathy after an 8-week vibration therapy program (24). Additionally, a review study by del Pozo-Cruz et al reported the positive effect of WBV (whole-body vibration) on gait, balance, proprioception, strength, and health-related life quality of patients with neurological conditions (25). Moreover, the study by Boucher et al investigated the short-term effects of five 1-minute sessions of vibration on the neuromuscular body response in patients with chronic back pain and in healthy individuals. The results suggested increased lumbar EMG (electromyography) activity after WBV (26). The findings of the study above showed a reduced RLS mean score after massage. Russell postulated that massage therapy is effective in decreasing the severity of symptoms of RLS with the reduction being greater in the warmer foot. The study recommended exercises and massaging with heat to treat RLS (27). Hashemi et al declared that a three-week massage program with lavender oil is effective in diminishing the symptoms of RLS in hemodialysis patients (6). Kumar et al also announced the efficacy of massage therapy in reducing back pain (28). Furlan et al showed that massage therapy significantly diminishes pain intensity and disability compared to acupuncture (29). A study by Hayes and Cox on the effect of five minutes of podiatric massaging in ICU patients, concluded that podiatric massaging potentially increases relaxation during a short-term intervention in ICU patients and recommended it as a proper intervention for decreasing stress (30). Nonetheless, some review studies like that of Lewis et al on the impact of massage therapy on musculoskeletal pain or (31), the review study by Ernst on the impact of massage therapy on pain reduction (32), and the study by Haraldsson et al on performance pain in patients with neck ache (33) did not approve the efficacy of massage in reducing musculo-skeletal pain, though they do not completely reject its efficacy by stating that on sufficient reasons for the use of massage therapy in decreasing musculo-skeletal pain was existed. The study by Metin and Ozdemir, aiming to investigate the comparative effect of massage aromatherapy and reflexology on pain and fatigue in patients with rheumatoid arthritis in three groups (the massage group $=17$ patients, knee massage for 30 minutes; plantar reflexology group $=17$ patients, podiatric massage for 40 minutes; and control group $=17$ individuals with no intervention) for 6 weeks. They concluded that both interventions can positively affect pain and fatigue in patients with rheumatoid arthritis. Of course, the reflexology intervention reduced pain and fatigue more significantly compared to aromatherapy (34). Lundeberg et al demonstrated that vibration with moderate speed is effective in decreasing acute or chronic musculo-skeletal pain (35).

Most studies on the effect of vibration and massage therapy approve the findings of the present study in effect that vibration and massage improve the symptoms of RLS. Our results revealed that vibration improved sleep quality in patients. The study by Burbank on the effect of vibration on the value of sleep in RLS patients found that RLS-related sleep disturbance significantly decreased after one month of vibration therapy (36). These findings are consistent with our results, while vibration therapy exerts a positive

Table 2. Comparison of severity of RLS symptoms before and after massage therapy and vibration therapy

\begin{tabular}{|c|c|c|c|c|}
\hline & Before intervention & After intervention & \multirow{2}{*}{ Differences in means } & \multirow{2}{*}{$\boldsymbol{P}$ value (Paired $t$ test) } \\
\hline & Mean \pm SD & Mean $\pm S D$ & & \\
\hline Massage & $18.99 \pm 3.292$ & $15.50 \pm 2.436$ & 3.49 & 0.0001 \\
\hline Vibration & $18.99 \pm 3.292$ & $12.82 \pm 2.726$ & 6.17 & 0.001 \\
\hline
\end{tabular}

Table 3. Comparison of sleep quality before and after massage therapy and vibration therapy

\begin{tabular}{lcccc}
\hline & Sleep quality before intervention & Sleep quality after intervention & \multirow{2}{*}{ Differences in means } & \multirow{P}{*}{ Mean value (Paired $t$ test) } \\
\cline { 2 - 4 } Massage & $10.16 \pm 3.96$ & $9.22 \pm 3.48$ & 0.94 & 0.001 \\
Vibration & $10.16 \pm 3.966$ & $8.43 \pm 3.19$ & 1.73 & 0.001
\end{tabular}


effect on improving the RLS symptoms. The results of another study revealed that massage therapy improved sleep quality. Furthermore, the study by Malekshahi et al demonstrated the efficacy of massaging on improving sleep and life quality of ESRD patients recommending this method as a non-invasive treatment for patients with sleep disorders (37). Moreover, the study by Unal and Akpin, exploring the effect of back massage and plantar reflexology on fatigue and sleep quality in hemodialysis patients indicated that these methods improved sleep quality and fatigue in hemodialysis patients, with plantar reflexology being more effective than lower back and upper back massage (20). Since the acquisition of these methods is rather simple and their performance is possible and cheap for almost all patients, they can be recommended as non-pharmaceutical methods to be used in reducing this syndrome which is common among hemodialysis patients. Therefore, the patients can readily master these procedures and perform them at home to enjoy the positive effects of vibration and massage therapy through increasing blood supply to muscles and reducing tension and anxiety to diminish the severity of RLS symptoms and improve their sleep quality to gain better efficiency. Patients may also be educated in performing these methods under supervision to reduce the annoying symptoms of this syndrome during hemodialysis. This will be a major step in decreasing the complications by the treatment team.

\section{Conclusion}

Our results suggested that vibration and massage therapy significantly reduced the mean intensity of RLS symptoms and improved sleep quality in hemodialysis patients.

\section{Limitations of the study}

One of the limitations of this study was the researcher's inability to blind the study as it was out of control. It is recommended that future studies be focused on comparing the effects of other methods of complementary medicine on sleep quality and severity of RLS symptoms in hemodialysis patients.

\section{Acknowledgments}

We would like to thank the Deputy-in-Research at Rafsanjan University of Medical Sciences for their support of this project. We also thank all the personnel in Hemodialysis Ward of the hospital, all the patients, and all the individuals who helped us in carrying out the study.

\section{Authors' contribution}

SA participated in research design, the writing of the paper, and the performance of the research. $\mathrm{HH}$ and MK contributed to the study design, preparation of the manuscript and final revision. AF acted as the consultant of study. All authors read and approved the paper.
Conflicts of interest

The authors declare no conflict of interest.

\section{Ethical considerations}

Ethical issues (including plagiarism, data fabrication, double publication) have been completely observed by the authors.

\section{Funding/Support}

This paper is extracted from an ICU Nursing MSc thesis of Somayeh Azimpour (Thesis \# 2016) in Rafsanjan Medical University.

\section{References}

1. Beladi-Mousavi SS, Jafarizade M, Shayanpour S, Bahadoram M, Moosavian SM, Houshmand G. Restless legs syndrome: associated risk factors in hemodialysis patients. Nephrourol Mon. 2015;7:e31967. doi: 10.5812/numonthly.31967

2. Kimmel PL, Peterson RA. Depression in end-stage renal disease patients treated with hemodialysis: tools, correlates, outcomes, and needs. Semin Dial. 2005;18:91-7. doi: 10.1111/j.1525-139X.2005.18209.X

3. Bag E, Mollaoglu M. The evaluation of self-care and self-efficacy in patients undergoing hemodialysis. J Eval Clin Pract. 2010;16:605-10. doi: 10.1111/j.13652753.2009.01214.X

4. Rangarajan S, D’Souza GA. Restless legs syndrome in Indian patients having iron deficiency anemia in a tertiary care hospital. Sleep Med. 2007;8:247-51. doi: 10.1016/j. sleep.2006.10.004

5. Abideen ZU, Mahmud SN, Mushtaq F, Farooq MU. Association of hemodialysis inadequacy and duration with restless legs syndrome: a cross sectional study. Cureus. 2018;10:e2570. doi: 10.7759/cureus.2570

6. Hashemi SH, Hajbagheri A, Aghajani M. The effect of massage with lavender oil on restless leg syndrome in hemodialysis patients: a randomized controlled trial. Nurs Midwifery Stud. 2015;4:e29617. doi: 10.17795/ nmsjournal29617.

7. Wali SO, Alkhouli AF. Restless legs syndrome among Saudi end-stage renal disease patients on hemodialysis. Saudi Med J. 2015;36:204. doi: 10.15537/smi.2015.2.10036

8. Leschziner G, Gringras P. Restless legs syndrome. BMJ. 2012;344:e3056. doi: 10.1136/bmj.e3056

9. Wong TY, Li TS, Ko FW, Hui DS, Choy DK, Szeto CC, et al. Prevalence of sleep disturbances in Chinese patients with end stage renal failureon maintenance hemodialysis. Med Sci Mon Bas Res. 2002;8:CR331-6.

10. Gade K, Blaschke S, Rodenbeck A, Becker A, AndersonSchmidt H, Cohrs S. uremic restless legs syndrome (RLS) and sleep quality in patients with end-stage renal disease on hemodialysis: potential role of homocysteine and parathyroid hormone. Kidney Blood Press Res. 2013;37(45):458-63. doi: 10.1159/000355727

11. Klingelhoefer L, Bhattacharya K, Reichmann H. Restless legs syndrome. Clin Med. 2016;16:379-82. doi: 10.7861/ clinmedicine.16-4-379

12. Aurora RN, Kristo DA, Bista SR, Rowley JA, Zak RS, Casey $\mathrm{KR}$, et al. The treatment of restless legs syndrome and 
periodic limb movement disorder in adults-an update for 2012 practice parameters with an evidence-based systematic review and meta-analyses: an American Academy of Sleep Medicine Clinical Practice Guideline. Sleep. 2012;35:103962. doi: 10.5665/sleep.1988.

13. Issurin V, Liebermann D, Tenenbaum G. Effect of vibratory stimulation training on maximal force and flexibility. J Sports Sci. 1994;12:561-6. doi: 10.1080/02640419408732206

14. Prisby RD, Lafage-Proust M-H, Malaval L, Belli A, Vico L. Effects of whole body vibration on the skeleton and other organ systems in man and animal models: what we know and what we need to know. Ageing Res Rev. 2008;7:319-29. doi: 10.1016/j.arr.2008.07.004

15. Sands WA, McNeal JR, Stone MH, Haff GG, Kinser AM. Effect of vibration on forward split flexibility and pain perception in young male gymnasts. Int J Sports Physiol Perform. 2008;3:469-81. doi: 10.1123/ijspp.3.4.469

16. Mitchell UH, Johnson PK. Vibration and skin blood flow changes in subjects with restless legs syndrome. J Parkinsonism Res. 2014;4:9-16. doi: 10.2147/JPRLS.S58556

17. Doyle A, Chalmers K, Chinn DJ, McNeill F, Dall N, Grant $\mathrm{CH}$. The utility of whole body vibration exercise in haemodialysis patients: a pilot study. Clin Kidney J. 2017;10:822-9. doi: 10.1093/ckj/sfx046

18. Field TM. Massage therapy effects. Am Psychol. 1998;53(12):1270. doi: 10.1037/0003-0066x.53.12.1270

19. Sullivan CM, Cain K, Theurer J, et al. Intradialytic massage for leg cramps among hemodialysis patients: a pilot randomized controlled trial. Int J Ther Massage Bodywork. 2016;9:3-8.

20. Unal KS, Akpinar RB. The effect of foot reflexology and back massage on hemodialysis patients' fatigue and sleep quality. Complement Ther in Clin Pract. 2016;24:139-44. doi: 10.1016/j.ctcp.2016.06.004

21. Molahosseini S, Mohammadzadeh S, Kamali P. Frequency of sleep disorder and restless legs syndrome in patients referring to hemodialysis units in university hospitals in Tehran in 2003. Med Sci J Islam Azad Univ-Tehran Med Branch. 2005;15:27-30

22. Farrahi J, Nakhaee N, Sheibani V, Garrusi B, Amirkafi A. Psychometric properties of the Persian version of the Pittsburgh Sleep Quality Index addendum for PTSD (PSQI-A). Sleep Breath. 2009;13:259. doi: 10.1007/s11325008-0233-3

23. Yoosefinejad AK, Shadmehr A, Olyaei G, Talebian S, Bagheri $\mathrm{H}$. The effectiveness of a single session of WholeBody Vibration in improving the balance and the strength in type 2 diabetic patients with mild to moderate degree of peripheral neuropathy: a pilot study. J Bodyw Mov Ther. 2014;18:82-6. doi: 10.1016/j.jbmt.2013.10.007

24. Junggi $H$. Whole body vibration therapy for diabetic peripheral neuropathic pain: A case report. Health Sci J. 2011;5:66-71

25. del Pozo-Cruz B, Adsuar JC, Parraca JA, del Pozo-Cruz J,
Olivares PR, Gusi N. Using whole-body vibration training in patients affected with common neurological diseases: a systematic literature review. J Altern Complement Med. 2012;18:29-41. doi: 10.1089/acm.2010.0691.

26. Boucher J-A, Abboud J, Dubois J-D, Legault E, Descarreaux $\mathrm{M}$, Henchoz Y. Trunk neuromuscular responses to a single whole-body vibration session in patients with chronic low back pain: a cross-sectional study. J Manipulative Physiol Ther. 2013;36:564-71. doi: 10.1016/j.jmpt.2013.09.001.

27. Russell M. Massage therapy and restless legs syndrome. J Bodyw Mov Ther. 2007;11:146-50. Doi: 10.1016/j. jbmt.2006.12.001

28. Kumar S, Beaton K, Hughes T. The effectiveness of massage therapy for the treatment of nonspecific low back pain: a systematic review of systematic reviews, Int J Gen Med. 2013;6:733-41. doi: 10.2147/ijgm.s50243

29. Furlan AD, Yazdi F, Tsertsvadze A. A systematic review and mata-analysis of efficacy, cost-effectiveness, and safety of selected complementary and alternative medicine for neck and low-back pain. Evid Based Complement Alternat Med. 2012;953139. doi: 10.1155/2012/953139

30. Hayes J, Cox C. Immediate effects of a five-minute foot massage on patients in critical care. Intensive Criti Care Nurs.15(2):77-82

31. Lewis M, Johnson MI. The clinical effectiveness of therapeutic massage for musculoskeletal pain: a systematic review. Physio. 2006;92:146-58. doi: 10.1016/j. physio.2006.02.008

32. Ernst E. Massage therapy for cancer palliation and supportive care: a systematic review of randomised clinical trials. Support Care Cancer. 2009;17:333-7. doi: 10.1007/ s00520-008-0569-Z

33. Haraldsson B, Gross A ,Myers CD, Ezzo J, Morien A, Goldsmith C, et al. Massage for mechanical neck disorders. Cochrane Database Syst Rev. 2006;3. Doi: 10.1002/14651858.CD004871.pub3

34. Metin ZG, Ozdemir L. The Effects of Aromatherapy Massage and Reflexology on Pain and Fatigue in Patients with Rheumatoid Arthritis: A Randomized Controlled Trial. Pain Manag Nurs. 2016;17:140-9. doi: 10.1016/j. pmn.2016.01.004

35. Lundeberg T, Nordemar R, Ottoson D. Pain alleviation by vibratory stimulation. Pain. 1984;20:25-44

36. Burbank F. Sleep improvement for restless legs syndrome patients. Part III: effect of treatment assignment belief on sleep improvement in restless legs syndrome patients. A mediation analysis. J Parkinsonism Res. 2013;3:23-9. doi: 10.2147/jprls.s40358

37. Malekshahi F, Aryamanesh F, Fallahi S. The effects of massage therapy on sleep quality of patients with endstage renal disease undergoing hemodialysis. J Clin Neuroscience Psychopath. 2018;20: 91-95. doi:10.5350/ sleep.Hypn.2017.19.0138.

Copyright (c) 2019 The Author(s); Published by Nickan Research Institute. This is an open-access article distributed under the terms of the Creative Commons Attribution License (http://creativecommons.org/licenses/by/4.0), which permits unrestricted use, distribution, and reproduction in any medium, provided the original work is properly cited. 